\title{
Towards a QCD equation of state with 2+1+1 flavors using the HISQ action
}

\section{A. Bazavov ${ }^{a}$, C. Bernard ${ }^{b}$, C. DeTar ${ }^{c}$, J. Foley $^{c}$, Steven Gottlieb ${ }^{d}$, Urs M. Heller ${ }^{*, e}$, , J.E. Hetrick ${ }^{f}$, J. Laiho ${ }^{g}$, L. Levkova ${ }^{c}$, J. Osborn ${ }^{h}$, R. Sugar ${ }^{i}$, D. Toussaint ${ }^{j}$, R.S. Van de Water ${ }^{k}$, and R. Zhou ${ }^{d}$}

${ }^{a}$ Department of Physics, Brookhaven National Laboratory, Upton, NY 11973, USA

${ }^{b}$ Department of Physics, Washington University, St. Louis, MO 63130, USA

${ }^{c}$ Department of Physics and Astronomy, University of Utah, Salt Lake City, UT 84112, USA

${ }^{d}$ Department of Physics, Indiana University, Bloomington, IN 47405, USA

${ }^{e}$ American Physical Society, One Research Road, Ridge, NY 11961, USA

${ }^{f}$ Physics Department, University of the Pacific, Stockton, CA 95211, USA

${ }^{g}$ SUPA, School of Physics \& Astronomy, University of Glasgow, Glasgow G12 8QQ, UK

${ }^{h}$ Argonne Leadership Computing Facility, Argonne National Laboratory, Argonne, IL 60439, USA

${ }^{i}$ Department of Physics, University of California, Santa Barbara, CA 93106, USA

${ }^{j}$ Department of Physics, University of Arizona, Tucson, AZ 85721, USA

${ }^{k}$ Theoretical Physics Department, Fermi National Accelerator Laboratory, Batavia, IL 60510, USA

${ }^{\dagger}$ E-mail: hellereaps.org

\section{MILC Collaboration}

\begin{abstract}
We present first, preliminary results for the QCD equation of state with $2+1+1$ flavors of highly improved staggered quarks (HISQ), that is, including the effects of a dynamical charm quark. This first study uses a line of constant physics (LCP) with the light-quark mass at $m_{l}=m_{s} / 5$ and physical strange- and charm-quark masses. With increasing temperature, the effects of the charm quark, as in earlier partially quenched studies, start to be visible at temperatures where the equation of state is becoming insensitive to the value of the light (up/down) quark mass.
\end{abstract}

The 30th International Symposium on Lattice Field Theory

June 24 - 29, 2012

Cairns, Australia

\footnotetext{
${ }^{*}$ Speaker.
} 


\section{Introduction}

Using (highly) improved staggered fermion actions two groups have brought the computation of the QCD equation of state (EoS) with 2+1 flavors (up/down and strange) within reach [1, 2]. Both groups use a tree-level improved gauge action. The Budapest-Wuppertal collaboration, Ref. [1], uses staggered fermions with stout smearing and the HotQCD collaboration, Ref. [2], uses the highly improved staggered quark (HISQ) action [3]. The HISQ action was designed to allow inclusion of a dynamical charm quark with controlled discretization errors. Indeed, the MILC collaboration has started an extensive zero-temperature simulation program with $2+1+1$ flavors, including dynamical charm, [円].

Since charm was certainly present in the evolution of the early Universe, it appears to make sense to include dynamical charm in the ab initio computations of the QCD EoS. A perturbative calculation indicates that the effects of charm become visible around $T \sim 350 \mathrm{MeV}$ [5], i.e., at a temperature only about twice the QCD crossover temperature $T_{c}$. Nonperturbative computations, with charm treated partially quenched with $2+1$ flavors of asqtad sea quarks, gave similar results [6]. The charm quark appears to start contributing to the EoS at temperatures reached in heavy ion collision experiments, in particular at the LHC. Thus, if charm thermalizes quickly enough, inclusion of charm in the EoS might even be important for the description of heavy ion collision experiments.

The Budapest-Wuppertal collaboration has began 2+1+1 flavor EoS computations with the stout staggered action [7]. Here we present preliminary results of the MILC collaboration using the HISQ action, which was designed, in part, to allow inclusion of dynamical charm with small discretization errors.

\section{Lattice setup}

The simulations for the present study use the same actions as MILC's zero-temperature $2+1+1$ flavor HISQ project [ $[$ ]. The gauge action is the one-loop Lüscher-Weisz action with tadpole improvement. It thus depends on the tadpole factor $u_{0}(\beta)=\left\langle\operatorname{Tr} U_{p} / 3\right\rangle^{1 / 4}$ which is determined during thermalization. For the fermions we use the highly improved staggered quark (HISQ) action [3]. For the charm quark, with $a m_{c}$ not very small, this includes a mass (and therefore gauge coupling) dependent correction factor $\varepsilon_{N}$ for the so-called Naik term in the action. This term insures that the charm quark has the correct, relativistic dispersion relation to good accuracy.

Here we compute the EoS along a line of constant physics (LCP) defined by $m_{l}=m_{s} / 5$, with $m_{s}$ and $m_{c}$ roughly tuned to the physical strange- and charm-quark masses, using the pion, kaon and spin-averaged charmonium ground state. The zero-temperature ensembles of Ref. [⿰亻 supplemented by, so far, five new ones for this thermodynamics project. Two, on coarser lattices than considered in Ref. [ $₫$ ], were used for the determination of the LCP, and three further ones are needed for zero-temperature subtractions. Prior to starting creation of an ensemble the strange- and charm-quark masses can only be estimated. Table 1 lists the ensembles used to define the LCP used. It also shows the a posteriori determined corrected strange- and charm-quark masses. $r_{1} \approx 0.31$ $\mathrm{fm}$, a parameter determined from the force between a static quark-anitquark pair by $r_{1}^{2} F\left(r_{1}\right)=1$, is used to set the lattice scale. 


\begin{tabular}{|l|l|l|l|l|l|c|l|l|}
\hline$\beta$ & $a m_{s}$ & $a m_{c}$ & $u_{0}$ & $\varepsilon_{N}$ & $a / r_{1}$ & size & $a m_{s}^{\text {corr }}$ & $a m_{c}^{\text {corr }}$ \\
\hline 5.4 & 0.091 & 1.339 & 0.83496 & -0.7995 & $0.708(12)$ & $16^{3} \times 48$ & $0.1058(30)$ & $1.3386(22)$ \\
5.6 & 0.0785 & 1.080 & 0.84577 & -0.5168 & $0.5828(27)$ & $16^{3} \times 48$ & $0.0799(6)$ & $1.1021(13)$ \\
5.8 & 0.065 & 0.838 & 0.85535 & -0.3582 & $0.4857(54)$ & $16^{3} \times 48$ & $0.0668(15)$ & $0.8472(16)$ \\
6.0 & 0.0509 & 0.635 & 0.86372 & -0.2308 & $0.3883(26)$ & $24^{3} \times 64$ & $0.0516(7)$ & $0.6363(9)$ \\
6.3 & 0.037 & 0.440 & 0.87416 & -0.1204 & $0.2858(20)$ & $32^{3} \times 96$ & $0.0367(5)$ & $0.4396(7)$ \\
6.72 & 0.024 & 0.286 & 0.88577 & -0.0533 & $0.1872(6)$ & $48^{3} \times 144$ & $0.0225(1)$ & $0.2768(3)$ \\
\hline
\end{tabular}

Table 1: The zero temperature lattices used to determine the line of constant physics with $m_{l}=m_{s} / 5$. Columns 2 and 3 give the simulation strange- and charm-quark masses and columns 6 and 7 the a posteriori determined corrected values. Columns 4 and 5 give the tadpole factor, $u_{0}$, and the Naik term, $\varepsilon_{N} . a / r_{1}$ is used the set the lattice scale.
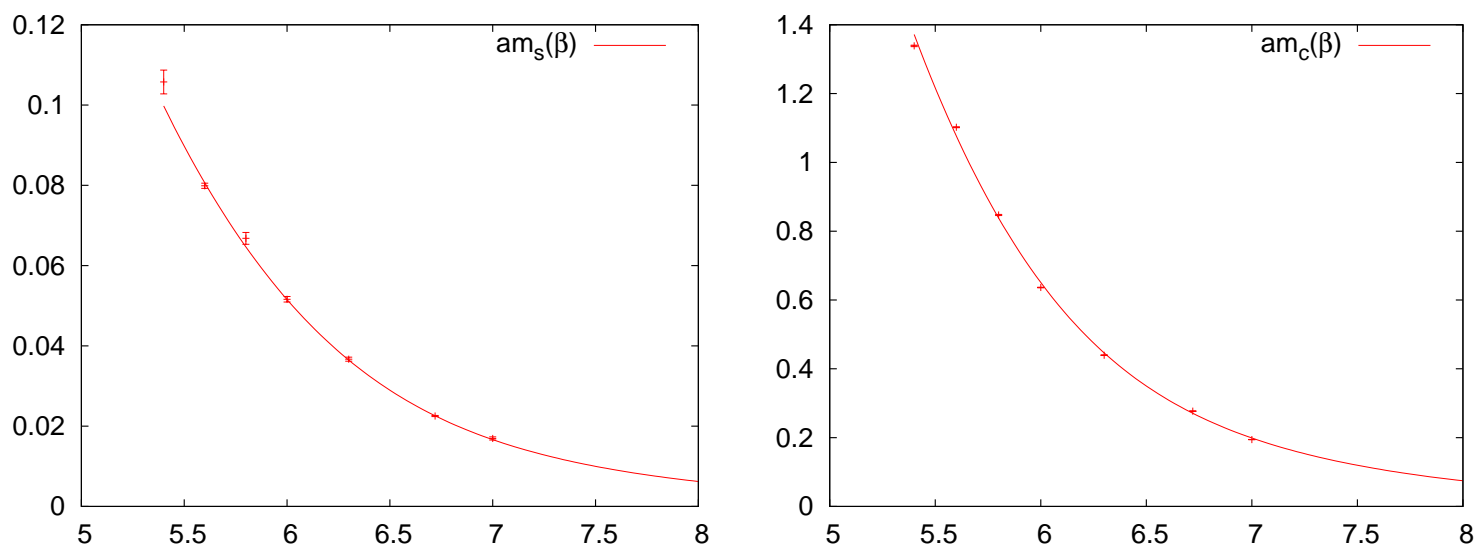

Figure 1: The bare strange- and charm-quark mass as a function of $\beta$ along the line of constant physics with $a m_{l} / a m_{s}=1 / 5$, and the fitted parametrizations of the form Eq. 2.1.

The bare strange- and charm-quark masses are shown in Fig. 1 together with a parametrization of the form

$$
\operatorname{am}_{q}(\beta)=\frac{c_{q}^{(0)} f(\beta)+c_{q}^{(2)}(10 / \beta) f^{3}(\beta)}{1+d_{q}^{(2)}(10 / \beta) f^{2}(\beta)}\left(\frac{20 b_{0}}{\beta}\right)^{4 / 9}
$$

for $q=s, c$, where

$$
f(\beta)=\left(\frac{10 b_{0}}{\beta}\right)^{-b_{1} /\left(2 b_{0}^{2}\right)} \exp \left(-\beta / 20 b_{0}\right)
$$

is the integrated perturbative two-loop $\beta$-function. ${ }^{1}$ The coefficients $c_{q}^{(0)}, c_{q}^{(2)}$ and $d_{q}^{(2)}$ were determined by a fit of (2.1) to the data. The lattice scale $r_{1}$, also listed in Table 1, is fit to a similar form,

$$
\left(r_{1} / a\right)(\beta)=\frac{c_{r}^{(0)} f(\beta)+c_{r}^{(2)}(10 / \beta) f^{3}(\beta)}{1+d_{r}^{(2)}(10 / \beta) f^{2}(\beta)} .
$$

\footnotetext{
${ }^{1}$ We have used the three-flavor $\beta$-function for this work. Switching to the four-flavor $\beta$-function is compensated by changes in the fit coefficients and has a negligible effect on the EoS results.
} 


\section{Ingredients to the equation of state}

The quantity convenient to compute on the lattice is the trace anomaly, or interaction measure, in units of $T^{4}$

$$
\frac{\Theta^{\mu \mu}(T)}{T^{4}} \equiv \frac{\varepsilon-3 p}{T^{4}}=T \frac{\partial}{\partial T}\left(p / T^{4}\right)
$$

with the pressure given by $p / T=V^{-1} \ln Z$. Integration of the trace anomaly along the LCP then gives the pressure.

Changes in the temperature $T=1 /\left(a N_{\tau}\right)$ come, at fixed $N_{\tau}$, from changes in the lattice spacing $a$ along the LCP, and are related to changes in the gauge coupling given by the $\beta$-function

$$
R_{\beta}(\beta)=T \frac{\mathrm{d} \beta}{\mathrm{d} T}=-a \frac{\mathrm{d} \beta}{\mathrm{d} a}=\left(r_{1} / a\right)(\beta)\left(\frac{\mathrm{d}\left(r_{1} / a\right)(\beta)}{\mathrm{d} \beta}\right)^{-1}
$$

We introduce similar $\beta$-functions describing the changes of bare quark masses, tadpole factor $u_{0}(\beta)$ and Naik-term correction factor for the charm quark, $\varepsilon_{N}(\beta)$, with the gauge coupling,

$$
R_{m_{q}}(\beta)=\frac{1}{a m_{q}(\beta)} \frac{\mathrm{d} a m_{q}(\beta)}{\mathrm{d} \beta} \quad \text { for } q=l, s, c,
$$

with $R_{m_{l}}=R_{m_{s}}$ along the considered LCP, and

$$
R_{u}(\beta)=\beta \frac{\mathrm{d} u_{0}(\beta)}{\mathrm{d} \beta}, \quad R_{\mathcal{\varepsilon}}(\beta)=\frac{\mathrm{d} \varepsilon_{N}(\beta)}{\mathrm{d} \beta} .
$$

To evaluate $R_{u}(\beta)$ we fitted $u_{0}$ as $u_{0}(\beta)=c_{1}+c_{2} \mathrm{e}^{-\mathrm{d}_{1} \beta} . R_{\varepsilon}$, finally, is obtained using a polynomial fit to the numerical values for $\varepsilon_{N}(\beta)$.

To normalize the interaction measure to zero at zero temperature we subtract observables computed on zero-temperature lattices from those computed on lattices with temporal extent $N_{\tau}$, defining

$$
\Delta(X)=\langle X\rangle_{\tau}-\langle X\rangle_{0}
$$

where the latter is the expectation value on the zero-temperature lattices at the same (bare) parameters. Thus we have

$$
\frac{\Theta^{\mu \mu}(T)}{T^{4}}=N_{\tau}^{4} R_{\beta}(\beta)\left\{-\Delta\left(s_{G}\right)-R_{u}(\beta) \Delta\left(\frac{\mathrm{d} s_{G}}{\mathrm{~d} u_{0}}\right)+\mathscr{I}_{F}\right\},
$$

where $s_{G}$ denotes the gauge action density and $\mathscr{I}_{F}$ indicates the "fermion terms":

$$
\mathscr{I}_{F}=R_{m_{s}}(\beta)\left[2 a m_{l} \Delta\left(\bar{\psi}_{l} \psi_{l}\right)+a m_{s} \Delta\left(\bar{\psi}_{s} \psi_{s}\right)\right]+R_{m_{c}}(\beta) a m_{c} \Delta\left(\bar{\psi}_{c} \psi_{c}\right)+R_{\varepsilon} \Delta\left(\bar{\psi}_{c}\left[\mathrm{~d} M_{c} / \mathrm{d} \varepsilon_{N}\right] \psi_{c}\right) .
$$

In the last term, $M_{c}$ denotes the charm-quark fermion matrix.

The needed $\left\langle\bar{\psi}_{q} \psi_{q}\right\rangle_{0, \tau}$ for $q=l, s, c$ and $\left\langle\bar{\psi}_{c}\left[\mathrm{~d} M_{c} / \mathrm{d} \varepsilon_{N}\right] \psi_{c}\right\rangle_{0, \tau}$ are evaluated using stochastic estimators. Because of the "rooting procedure" they are the contribution from a single taste, i.e., with a factor $1 / 4$ implied.

We have generated data from finite-temperature simulations with $N_{\tau}=6,8,10$, and 12, which should allow us, with sufficient statistics, to perform a controlled continuum extrapolation. The 


\begin{tabular}{|l|l|r|r|r|r|r|r|r|r|}
\hline & \multicolumn{2}{|c|}{$N_{\tau}=6$} & \multicolumn{2}{c|}{$N_{\tau}=8$} & \multicolumn{2}{c|}{$N_{\tau}=10$} & \multicolumn{2}{c|}{$N_{\tau}=12$} & $T=0$ \\
\hline$\beta$ & $T$ & TU & $T$ & TU & $T$ & TU & $T$ & TU & TU \\
\hline 5.400 & 163 & 10,990 & & & & & & & 5,295 \\
5.600 & 182 & 10,990 & 136 & 10,990 & & & & & 5,295 \\
5.800 & 218 & 10,990 & 163 & 10,990 & & & & & 5,300 \\
6.000 & 272 & 10,990 & 204 & 10,990 & 163 & 10,990 & 136 & 12,990 & 5,560 \\
6.180 & & & 247 & 11,990 & 197 & 8,900 & 164 & 6,290 & 2,195 \\
6.300 & 369 & 10,990 & 277 & 10,990 & 222 & 10,990 & 185 & 10,990 & $\sim 6,000$ \\
6.530 & & & 352 & 11,990 & 282 & 9,610 & 235 & 10,990 & 3,180 \\
6.720 & 567 & 10,990 & 425 & 10,990 & 340 & 10,990 & 283 & 10,990 & 6,720 \\
6.875 & & & & & & & 329 & 11,990 & 3,374 \\
\hline
\end{tabular}

Table 2: Finite-temperature ensembles with temperature values in $\mathrm{MeV}$ and statistics in time units (TU). The last column gives the statistics in time units for the corresponding zero-temperature lattices used for the zero-temperature subtractions.

statistics assembled on the finite-temperature ensembles so far are listed in Table 2. Also given in the table are the statistics assembled for the zero-temperature subtractions, Eq. (3.5). Three new ensembles are being generated, in addtition to those listed in Table 1, i.e., the ones with, so far, less than 4000 TUs.

The contributions to the trace anomaly from the gauge action, including from the variation of the tadpole factor $u_{0}$, and from the light-, strange- and charm-quark condensates are shown in Fig. 2 . The contributions from the gauge action and the light- and strange-quark condensates peak around $T=200 \mathrm{MeV}$, with the strange-quark condensate peaking at a slightly higher temperature than the light-quark condensate. The contribution from the charm-quark condensates sets in more gradually than the other contributions, and at somewhat higher temperatures. Within the temperature range explored so far, up to about $550 \mathrm{MeV}$, we do not observe a clear peak.

The contribution due to the variation of the charm-quark Naik term has, so far, been computed only at two temperatures, $T=272 \mathrm{MeV}$ and $T=369 \mathrm{MeV}$, on $N_{\tau}=6$ lattices and the corresponding zero-temperature ensembles. As expected, the contribution to the trace anomaly is small, $-0.028(5)$ and $-0.067(4)$, respectively, less than $2 \%$ of the other contributions combined, and well within their statistical errors.

\section{The preliminary EoS}

The current, preliminary status of the trace anomaly with $2+1+1$ flavors is shown in Fig. 3 (left) for the various $N_{\tau}$. The contribution for the variation of the Naik term, found to be negligible for the two points where we have computed it so far, is not included. No continuum extrapolation has been attempted yet. In Fig. 3 (right) we compare the EoS with and without the valence charmquark contribution from the condensate. Both versions, of course, account for the effect of the sea-charm quarks. 

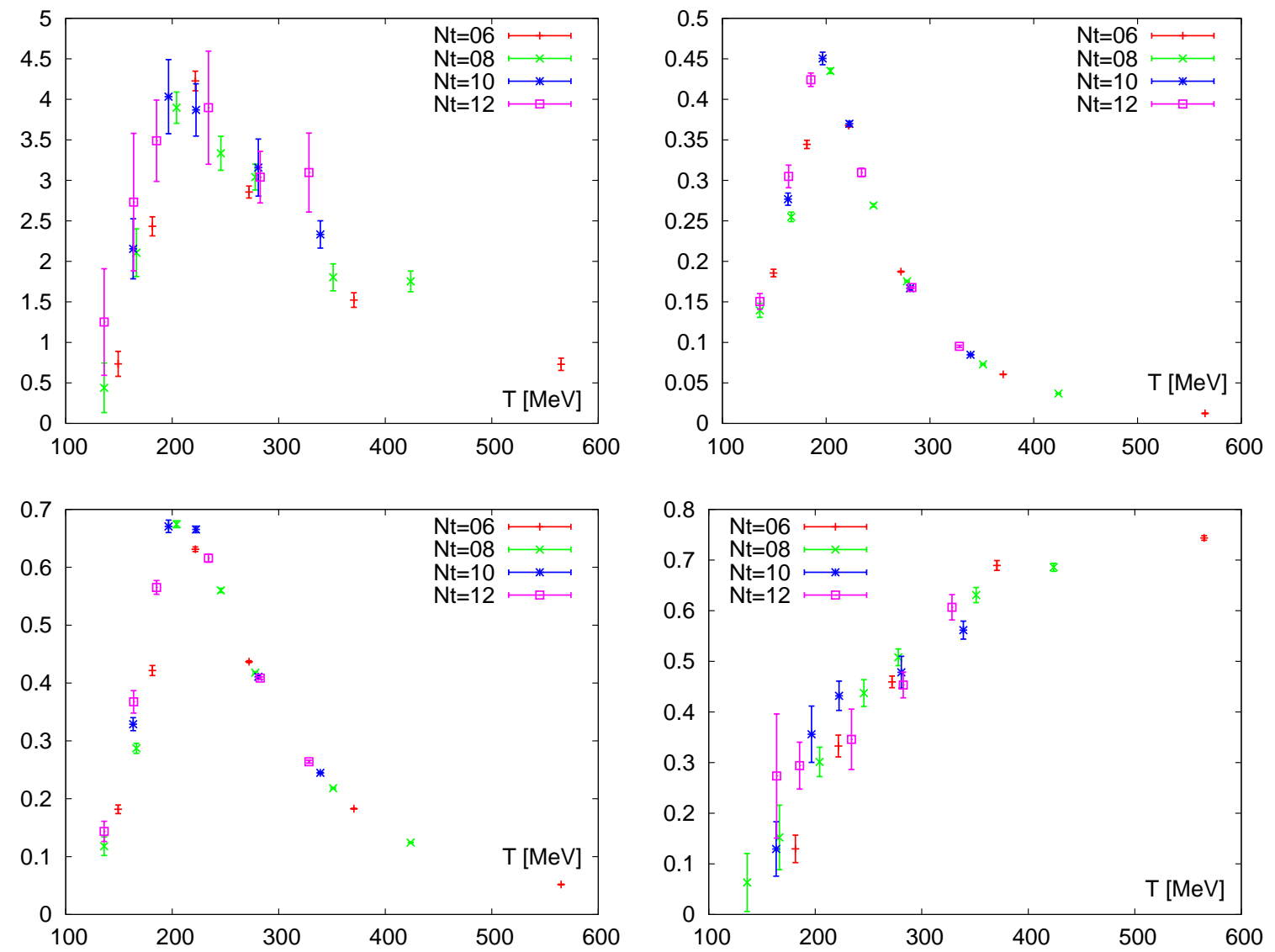

Figure 2: The contributions to the EoS from the gauge part of the action, i.e., the first two terms in Eq. 3.6) (top left), the light-quark condensate (top right), the strange-quark condensate (bottom left) and the charmquark condensate (bottom right).
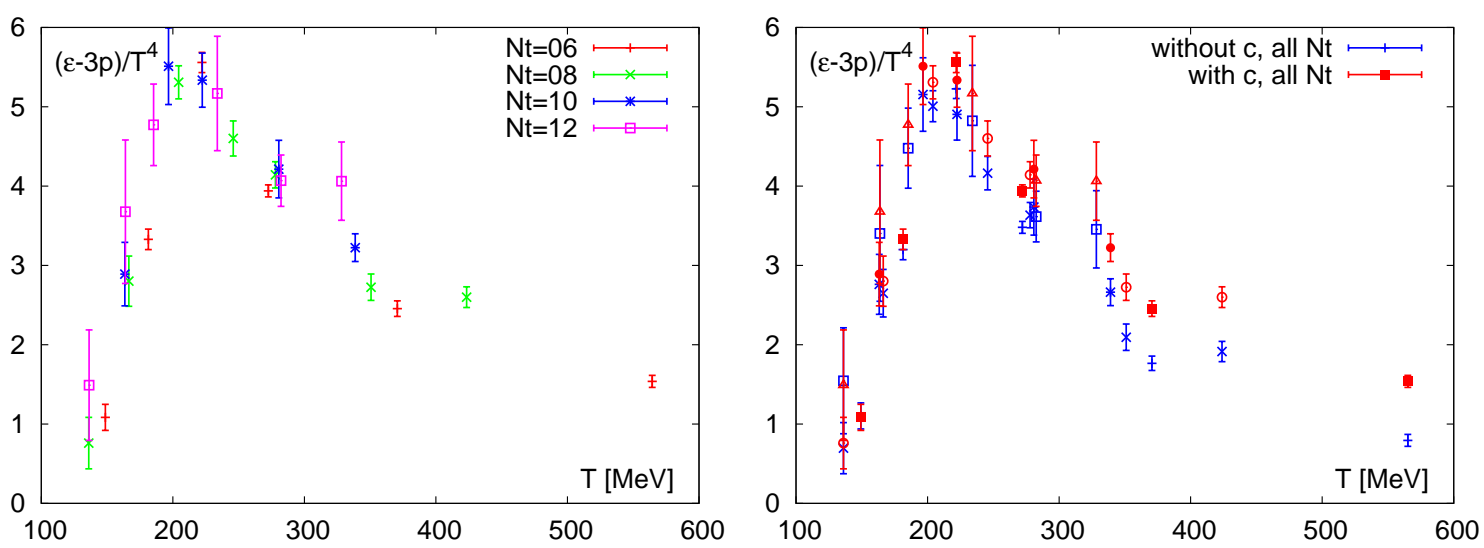

Figure 3: The current status of the EoS including the charm-quark contribution (left) and comparison of the EoS with and without the valence charm-quark contribution (right). 


\section{Conclusion and outlook}

The MILC collaboration has started a study of the QCD EoS with $2+1+1$ flavors, including a dynamical charm quark, using the HISQ action. The first study uses an LCP with $m_{l} / m_{s}=1 / 5$ with physical strange and charm sea-quark masses. We have preliminary results for finite temperature lattices with $N_{\tau}=6,8,10$, and 12 , but have not attempted a continuum extrapolation yet.

The effect of the charm quark on the EoS, as determined from the valence charm-quark contribution, sets in very gradually compared with the contributions from the gluons and light and strange quarks. It appears to become significant only at temperatures above the peak in the trace anomaly. At those temperatures, the value of the light-quark mass has a negligible influence on the trace anomaly. Thus, the result for the LCP with $m_{l} / m_{s}=1 / 5$ studied here should be close to that in the case of physical light-quark masses. The present study will be completed, with a proper continuum limit, in the near future.

\section{Acknowledgments}

Computations for this work were carried out with resources provided by the USQCD Collaboration, the Argonne Leadership Computing Facility, and the National Energy Research Scientific Computing Center, which are funded by the Office of Science of the U.S. Department of Energy; and with resources provided by the National Center for Atmospheric Research, the National Institute for Computational Science, the Pittsburgh Supercomputer Center, the San Diego Supercomputer Center, and the Texas Advanced Computer Center, which are funded through the National Science Foundation's XSEDE Program. This work was supported in part by the U.S. Department of Energy under Grants DE-FG02-91ER-40628, DE-FG02-91ER-40661, DE-FG0204ER-41298, and DE-FC02-06ER41446; and by the National Science Foundation under Grants PHY07-57333, PHY07-03296, PHY07-57035, PHY09-03571, PHY09-70137, and PHY10-67881. This manuscript has been co-authored by an employee of Brookhaven Science Associates, LLC, under Contract No. DE-AC02-98CH10886 with the U.S. Department of Energy. Fermilab is operated by Fermi Research Alliance, LLC, under Contract No. DE-AC02-07CH11359 with the U.S. Department of Energy.

\section{References}

[1] S. Borsanyi et al., JHEP 1011 (2010) 077 [arXiv:1007.2580].

[2] Peter Petreczky (HotQCD Collaboration), these proceedings, PoS (Lattice 2012) 069 .

[3] E. Follana et al. (HPQDD/UKQCD Collaboration), Phys. Rev. D 75 (2007) 054502 [arXiv:hep-lat/0610092].

[4] A. Bazavov et al. (MILC Collaboration), Phys. Rev. D 82 (2010) 074501 [arXiv:1004.0342]; and in preparation.

[5] M. Laine, Y. Schröder , Phys. Rev. D 74 (2006) 085009 [arXiv:hep-ph/0603048].

[6] C. DeTar et al. (MILC Collaboration), Phys. Rev. D 81 (2010) 114504 [arXiv:1003.5682].

[7] S. Borsanyi et al., PoS (Lattice 2011) 201] [arXiv:1204.0995]; and S. Krieg et al., these proceedings, PoS (Lattice 2012) 070 . 